\title{
Serrated polyps and polyposis of the colon: a brief review for surgeon endoscopists
}

Eric Hyun, MD, PhD

Ramzi M. Helewa, MD, MSc

Harminder Singh, MD, MPH

H. Robert Wightman, MD

Jason Park, MD, MEd

Accepted November 10, 2020

\section{Correspondence to:}

J. Park

St Boniface General Hospital

Z3031 - 409 Tache Ave

Winnipeg MB R2H $2 \mathrm{~A} 6$

jpark@sbgh.mb.ca

Cite as: Can J Surg 2021 November 2; 64(6). doi: $10.1503 /$ cjs. 018820

\section{SUMmARY}

Serrated polyps (SPs) were once considered benign, clinically unimportant lesions. However, it is now recognized that through the serrated neoplasia pathway (SNP), SPs play a role in the development of $15 \%-30 \%$ of cases of colorectal cancers (CRC). Furthermore, a high proportion of postcolonoscopy CRCs are believed to arise from SNP. Serrated polyps are classified into hyperplastic polyps, sessile serrated lesions, sessile serrated lesions with dysplasia, traditionally serrated adenomas, and unclassified serrated adenoma, each with a distinct morphological and molecular profile. Despite improved understanding, SPs remain a clinical challenge owing to evolving terminology, frequent pathologic misclassification, endoscopic underdetection, and high rates of incomplete removal. Surgeon endoscopists and surgeons who perform colorectal procedures will undoubtedly come across patients with SPs, and this paper summarizes some of the clinical challenges they will encounter. We also discuss the diagnosis and management of patients with serrated polyposis syndrome (SPS).

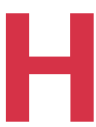

istorically, all serrated polyps (SPs) were thought to have no malignant potential. However, It is now recognized that up to $30 \%$ of sporadic colorectal cancers (CRCs) may arise from SPs via the serrated neoplasia pathway (SNP), a distinct carcinogenesis pathway separate from the traditional adenoma-carcinoma sequence. ${ }^{1}$

Despite their importance, the diagnosis and management of SPs remain challenging for several reasons. First, the terminology for SPs continues to evolve, which can lead to confusion with regards to malignant potential and management. Second, because SPs are difficult to visualize on colonoscopy, endoscopic detection and removal rates can be poor. Third, there can be wide variations in the pathologic interpretation of SPs owing to subtle differences in histomorphologic characteristics.

Finally, as understanding of the natural history of SPs continues to evolve, surveillance guidelines are inconsistent among organizations and are largely based on low-quality evidence.

It is imperative that surgeon endoscopists and surgeons who perform colorectal procedures stay up to date with the current literature regarding SPs, as they will undoubtedly care for patients with SPs. Here we discuss the diagnosis and management of SPs, organized by specific clinical challenges that surgeons may face. Key practice points are summarized in Box 1. We also provide a brief overview of serrated polyposis syndrome (SPS).

\section{Confusing TERMINOLOGY}

The term "serrated" describes a sawtooth folding of the crypt epithelium found in SPs (Figure 1). In the past, all SPs were classified as hyperplastic polyps (HPs) and were thought to have no malignant potential. However, subsequent studies described HPs with dysplasia and used the term "serrated adenoma." These HPs with dysplasia were later renamed "traditional 


\section{Box 1. Key practice points.}

- The World Health Organization (WHO) classification includes 5 subtypes of serrated polyps: HP, SSL, SSL with dysplasia, TSA, and serrated adenoma, unclassified.

- SSL has replaced the terms "sessile serrated adenoma" or "sessile serrated polyp" because SSLs may not have dysplasia on histology nor be polypoid in morphology.

- There are considerable histologic similarities between HPs and SSLs, which can lead to misdiagnosis. Right-sided HPs or those $>5 \mathrm{~mm}$ have higher odds of being reclassified as SSLs on re-review.

- It is recommended that all SPs be completely removed during colonoscopy, except for small ( $<5 \mathrm{~mm}$ ) multiple lesions in the sigmoid or rectum that are suspected to be HPs.

- Most current guidelines recommend a surveillance interval of 3 years for SSLs $>10 \mathrm{~mm}$ or with dysplasia, $>3 \mathrm{SSLs}<10 \mathrm{~mm}$, or TSAs of any size.

- Individuals with SPS have an increased risk of CRC and should undergo annual surveillance.

- Surgery is indicated for patients with SPS when polyps cannot be managed by endoscopic intervention or when CRC is diagnosed. The optimal extent of surgery is open to debate.

$\mathrm{CRC}=$ colorectal cancer; $\mathrm{HP}=$ hyperplastic polyp; $\mathrm{SP}=$ serrated polyp; $\mathrm{SPS}=$ serrated polyposis syndrome; $\mathrm{SSL}=$ sessile serrated lesion; $\mathrm{TSA}=$ traditional serrated adenoma.

serrated adenomas" (TSAs), to distinguish them from other, more common subtypes of SPs.

In 2019, the World Health Organization (WHO) proposed 5 subtypes of serrated polyps: HPs, microvesicular type or goblet cell-rich type; sessile serrated lesion (SSL); sessile serrated lesion with dysplasia (SSLD); TSA; and serrated adenoma, unclassified. ${ }^{1}$ Although the histopathological features of HP subtypes have been well studied, there is no obvious impact of this distinction on clinical management. With regards to SSLs, it is important to note that term has replaced the former terms "sessile serrated adenoma" (SSA) and "sessile serrated polyp" (SSP); endoscopists will now see SSL being used on their pathology reports. Experts discourage use of "SSA," because "adenoma" intrinsically implies presence of dysplasia, which SSLs may not necessarily have. The term "SSP" is also inaccurate since most SPs are flat rather than have "polypoid" morphology. An additional subtype of serrated adenoma unclassified is reserved to describe dysplastic polyps that are difficult to distinguish as either SSLD or TSA.

\section{DifFICULTY IN HISTOPATHOLOGIC INTERPRETATION}

Serrated polyps are differentiated by architectural characteristics rather than by nuclear changes, which characterize traditional dysplasia seen in adenomatous polyps (Figure 1).

True HPs show no or minimal cytologic atypia. They are considered benign with no significant malignant potential. In contrast, approximately $15 \%$ of SSLs show dysplastic features and, as such, SSLs are considered premalignant lesions. The pathologist will usually make explicit mention of dysplasia within an SSL in their report when it is present. Although dysplasia can be graded as low or high in SSLs, the clinical significance of this distinction is

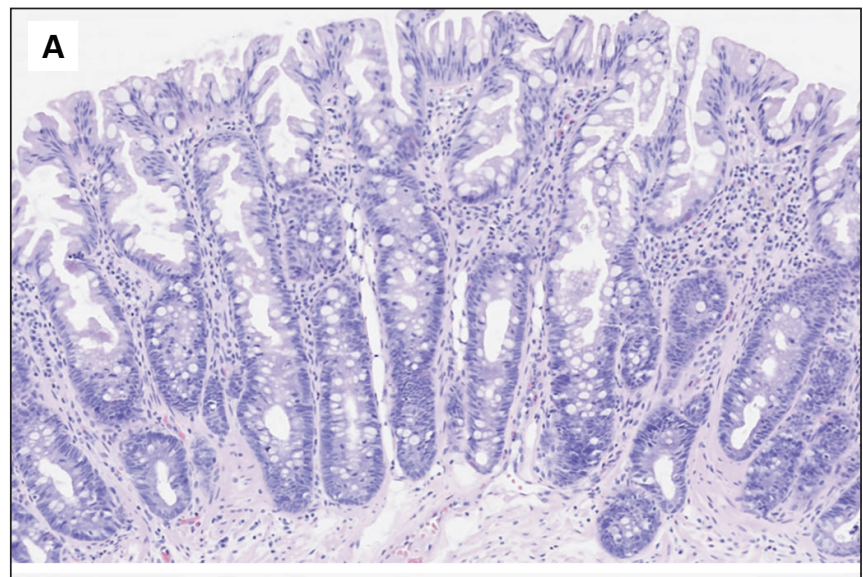

B

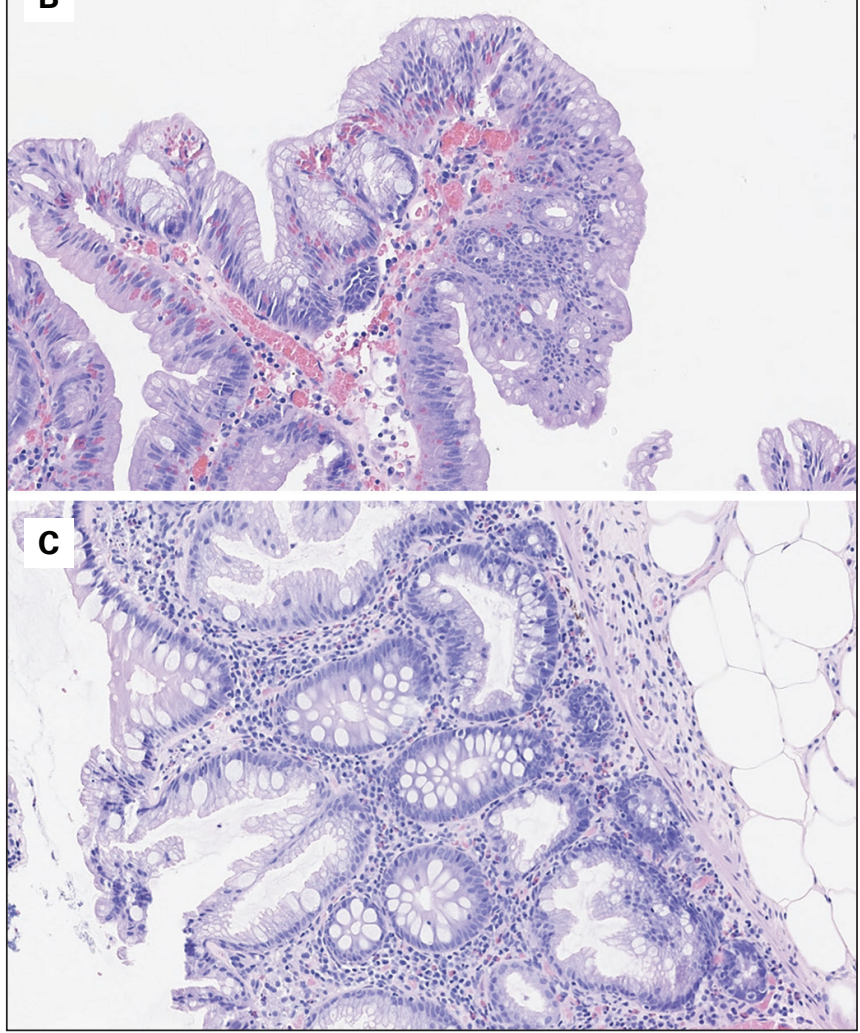

Fig. 1. Histopathologic features of serrated polyps. (A) Hyperplastic polyp, characterized by straight crypts that extend from the surface of the polyp to the muscularis propria. (B) Sessile serrated lesion, characterized by deep crypt dilation/serration with the horizontal extension of crypt bases (with shapes described as boot, $L$ or inverted $T$ ), and crypt herniation through the muscularis propria. (C) Traditional serrated adenoma, characterized by pencillate nuclei, eosinophillic cytoplasm and ectopic crypts.

unknown. Therefore, any presence of dysplasia in SSLs (SSLDs) is managed like high-grade dysplasia in the conventional adenomatous polyp. Traditional serrated adenomas have low-grade dysplasia by definition, and only highgrade dysplasia is reported separately.

There are considerable histologic similarities between HPs and SSLs, which can lead to misclassification. A 


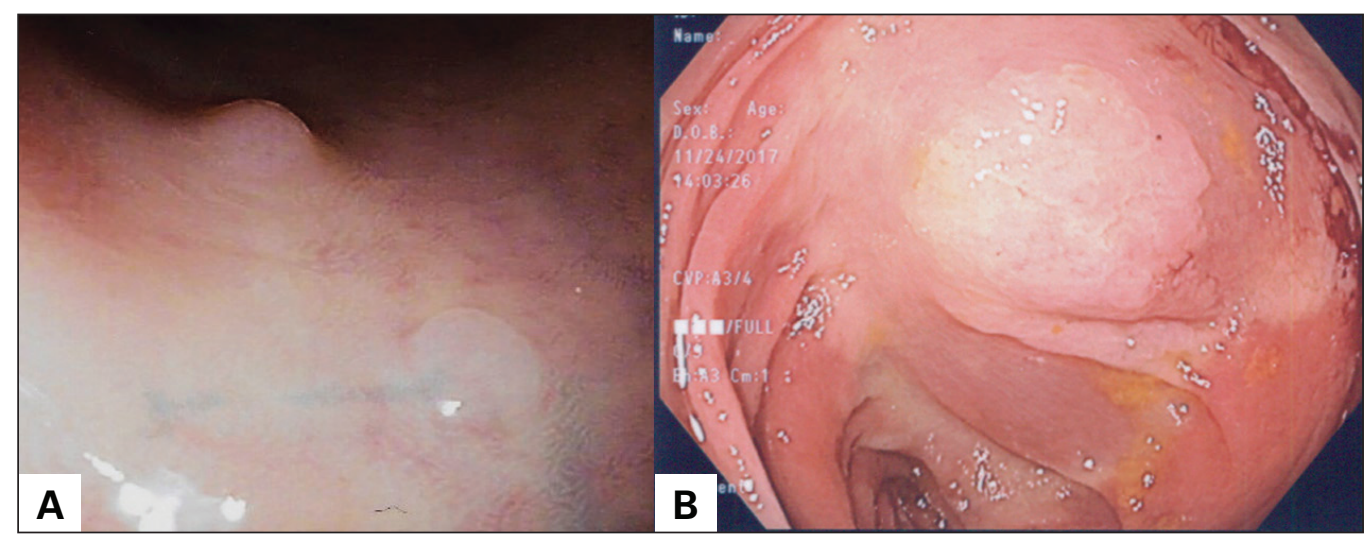

Fig. 2. Endoscopic appearance of serrated polyps. (A) Hyperplastic polyp, characterized by pale, smooth and flat morphology. (B) Sessile serrated lesion, characterized by pale and flat morphology with irregular borders, and commonly covered by a mucous cap.

Canadian study reviewed 4096 pathology reports and reclassified $17 \%$ of proximal HPs and $20 \%$ of HPs larger than $5 \mathrm{~mm}$ in size as SSLs. ${ }^{2}$ Location in the proximal colon and size larger than $5 \mathrm{~mm}$ were independently associated with HPs being reclassified as SSLs. This study reinforces the high level of caution needed when diagnosing or interpreting findings of any proximal and/or large SPs. It further underscores the importance of clear communication between endoscopists and pathologists regarding the location and size of any excised polyps on pathology requisition forms.

\section{LOW ENDOSCOPIC DETECTION AND REMOVAL RATES}

The endoscopic features of HPs include small size (usually $<5 \mathrm{~mm}$ ); pale, smooth appearance; and sessile morphology (Figure 2). Sessile serrated lesions are commonly found in the right colon and are pale with flat morphology and irregular borders (Figure 2). Because of excessive mucin production, most SSLs are covered with a thin mucous cap with a rim of debris. Traditional serrated adenomas have either sessile or pedunculated morphology with a pine-cone-like appearance.

\section{Endoscopic detection}

Many SPs are challenging to detect with endoscopy given their flat morphology, pale colour (which merges with the surrounding mucosa), indistinct border, and mucous covering. The endoscopic SP detection rate (SDR) has been shown to vary widely, even among expert endoscopists, raising a concern that many premalignant SPs are being underdetected. Kahi and colleagues showed that among 15 endoscopists the SDR varied from $1 \%$ to $18 \%$, which was far greater than the variability in adenoma detection rates (ADRs) among the same endoscopists. ${ }^{3}$ Although ADR for screening colonoscopy is an important quality indicator, there is no clear benchmark for SDR. It is possible that SDR will become a colonoscopy quality measure on its own or in conjunction with $\mathrm{ADR}$ in the future.
As SPs are easily missed on endoscopy, many studies have assessed additional endoscopy modalities, including chromoendoscopy and narrow-band imaging (NBI), to improve SDR. Pan-colonic chromoendoscopy is associated with a higher SDR than conventional colonoscopy, but the additional time required for this technique limits its use in routine screening. ${ }^{4}$ In contrast, NBI surveillance has not been found to decrease SP miss rates.

\section{Endoscopic removal}

Given the potential for malignant transformation, it is recommended that all SPs be completely removed during colonoscopy, except for small $(<5 \mathrm{~mm})$, multiple lesions in the sigmoid or rectum that are suspected to be HPs. Multiple diminutive rectosigmoid lesions should still be biopsied randomly to rule out more advanced SPs.

Serrated polyps smaller than $10 \mathrm{~mm}$ can generally be removed using standard snare polypectomy techniques. However, as SSLs have flat morphology with indistinct borders, they are more prone to incomplete resection than conventional adenomas. For SPs larger than $10 \mathrm{~mm}$, endoscopic mucosal resection (EMR) involving submucosal saline injection to lift the lesion followed by snare polypectomy with electrocautery is safe and effective.

Submucosal injection of methylene blue before polypectomy may help endoscopists better visualize the borders of SPs to ensure their complete removal (Figure 3).

\section{Alternative methods for detecting serrated polyps}

Aside from colonoscopy, other screening modalities for CRC include stool tests and computed tomography colonography (CTC), and have limited use for SP detection. A study involving asymptomatic patients undergoing concurrent fecal immunochemical test (FIT) and colonoscopy, showed a poor SDR for FIT, even for large SSLs. ${ }^{5}$ Accurate visualization of SPs during CTC is challenging owing to their flat morphology. A randomized trial 

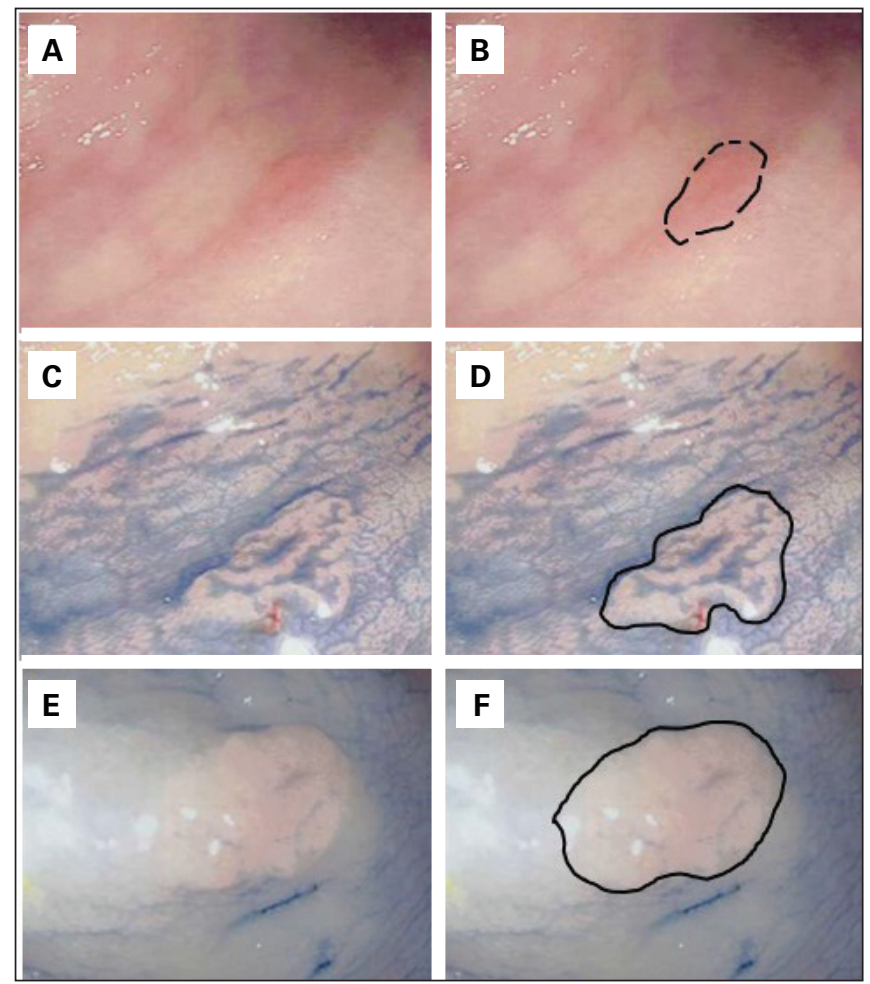

Fig. 3. Endoscopic appearance of serrated polyp (SP) before and after the use of methylene blue solution. (A) Endoscopic appearance of SP with indistinct border. (B) Border of SP depicted with black dotted lines. (C) Border of SP becomes more distinct after application of methylene blue on top of the polyp. (D) Border of SP after methylene blue application defined with a solid black line. (E) Border of SP can be more distinct and easier to snare after intramucosal injection of methylene blue solution. (F) Border of SP after intramucosal injection, noted with a solid black line.

involving 8844 patients showed that colonoscopy had a significantly higher SSL detection rate than CTC $(4.3 \%$ v. $0.8 \%) .{ }^{6}$ Although CTC is commonly performed after an incomplete colonoscopy, it is important to be aware of its limitation in detecting SPs.

\section{Surveillance guidelines based on weak evidence}

Multiple societies and expert panels have published guidelines for follow-up intervals for SPs (Table 1). However, current guidelines are based on weak evidence, as there is limited knowledge regarding "dwell time" or how long it takes for premalignant SPs to become CRC.

Most current guidelines recommend a surveillance interval of 3 years for patients with SSLs $10 \mathrm{~mm}$ or larger or with dysplasia, for patients with 3 or more SSLs smaller than $10 \mathrm{~mm}$, or for patients with TSAs of any size, which is consistent with the recommendations for high-risk or advanced adenomas. Canadian and American guidelines also recommend a surveillance interval of 5 years for patients with fewer than 3 SSLs smaller than $10 \mathrm{~mm} .{ }^{7,8}$

There are inconsistencies among guidelines for HPs, particularly for large or right-sided HPs. The US Multi-Society
Task Force on Colorectal Cancer guideline recommends a screening interval of 10 years for individuals with HPs and no other synchronous lesions, consistent with average-risk populations. ${ }^{8}$ On the other hand, the Canadian Association of Gastroenterology guidance makes no specific reference to HP surveillance guidelines at all. ${ }^{7}$ However, as previously discussed, approximately $20 \%$ of HPs located in the proximal colon and/or larger than $5 \mathrm{~mm}$ have been shown to be SSLs when re-examined by an expert pathologist. ${ }^{2}$ Findings such as these have led other groups to recommend more aggressive surveillance, particularly for large HPs. A primarily USbased expert panel considered large (>10 mm) HPs as intermediate risk and recommended 5-year surveillance intervals for these lesions. ${ }^{9}$ Some experts endorse that all SPs proximal to the sigmoid colon and all SPs of the rectosigmoid colon larger than $5 \mathrm{~mm}$ should be considered equivalent to SSL and be removed. Given the greater chance for histopathological misdiagnosis, when proximal HPs larger than $5 \mathrm{~mm}$ are diagnosed, the endoscopist should consult with the pathologist when making surveillance decisions.

\section{Serrated polyposis syndrome}

Serrated polyposis syndrome is characterized by the appearance of multiple SPs throughout the colon. In 2019, the WHO updated their clinical criteria for the diagnosis of SPS (Table 2).

Individuals with SPS have a higher risk of CRC, with some studies reporting a $40 \%-50 \%$ lifetime risk. Firstdegree relatives of patients with SPS are also at increased risk for both SPS and CRC. Given the increased risk for CRC, patients with SPS with intact colons are generally recommended to undergo annual surveillance (Table 1). A screening colonoscopy is recommended for first-degree relatives starting at age 40 years or at 10 years younger than the age at diagnosis of the youngest affected relative, whichever is earlier. Surgery is indicated for patients with SPS when polyps cannot be managed by endoscopic intervention or when CRC is diagnosed. There is, however, a paucity of data regarding the surgical management of patients with SPS. A study by Edelstein and colleagues reported that with follow-up of on average 5.3 years, 12 of $44(27 \%)$ patients with SPS underwent surgery..$^{10}$ Of these patients, $8(66 \%)$ underwent colectomy with ileorectal anastomosis (IRA) and 6 (33\%) underwent right hemicolectomy. Interestingly, during postoperative surveillance, 4 of 7 (57\%) were found to have SSLs or adenomas in the remaining colon/rectum (mean 2.5 yr). Long-term oncologic or survival outcomes were not reported.

There is no clear consensus on the optimal extent of surgery required for individuals with SPS. At the very least, surgical resection requires resection of affected segments harbouring lesions that are not amenable for endoscopic removal (to normal colon or at least a point of minimal disease that can be managed endoscopically). 
Content licence: This is an Open Access article distributed in accordance with the terms of the Creative Commons Attribution (CC BYNC-ND 4.0) licence, which permits use, distribution and reproduction in any medium, provided that the original publication is properly cited, the use is noncommercial (i.e., research or educational use), and no modifications or adaptations are made. See: https://creativecommons. org/licenses/by-nc-nd/4.0/

\section{References}

1. Pai RK, Makinen MJ, Rosty C. Colorectal serrated lesions and polyps. In: Board TWCoTE, ed. The WHO Classification of Tumours: Digestive System Tumours. 5th ed. Lyon (France): World Health Organization; 2019:163 to 9.

2. Singh H, Bay D, Ip S, et al. Pathological reassessment of hyperplastic colon polyps in a city-wide pathology practice: implications for polyp surveillance recommendations. Gastrointest Endosc 2012;76:1003-8.

3. Kahi CJ, Li X, Eckert GJ, et al. High colonoscopic prevalence of proximal colon serrated polyps in average-risk men and women. Gastrointest Endosc 2012;75:515-20.

4. Pohl J, Schneider A, Vogell H, et al. Pancolonic chromoendos- copy with indigo carmine versus standard colonoscopy for detection of neoplastic lesions: a randomised two-centre trial. Gut 2011;60:485-90.

5. Chang LC, Shun CT, Hsu WF, et al. Fecal immunochemical test detects sessile serrated adenomas and polyps with a low level of sensitivity. Clin Gastroenterol Hepatol 2017;15:872-9 e1.

6. IJspeert JEG, Tutein Nolthenius CJ, Kuipers EJ, et al. CTcolonography vs. colonoscopy for detection of high-risk sessile serrated polyps. Am 7 Gastroenterol 2016;111:516-22.

7. Leddin D, Enns R, Hilsden R, et al. Colorectal cancer surveillance after index colonoscopy: guidance from the Canadian Association of Gastroenterology. Can 7 Gastroenterol 2013;27:224-8.

8. Lieberman DA, Rex DK, Winawer SJ, et al. Guidelines for colonoscopy surveillance after screening and polypectomy: a consensus update by the US Multi-Society Task Force on Colorectal Cancer. Gastroenterology 2012;143:844-57.

9. Rex DK, Ahnen DJ, Baron JA, et al. Serrated lesions of the colorectum: review and recommendations from an expert panel. $\mathrm{Am} \mathcal{F}$ Gastroenterol 2012;107:1315-29; quiz 4, 30.

10. Edelstein DL, Axilbund JE, Hylind LM, et al. Serrated polyposis: rapid and relentless development of colorectal neoplasia. Gut 2013;62:404-8. 